BULLETIN OF THE

AMERICAN MATHEMATICAL SOCIETY

Volume 78, Number 5, September 1972

\title{
FOURIER COEFFICIENTS OF CERTAIN EISENSTEIN SERIES ${ }^{1}$
}

\author{
BY MARTIN L. KAREL
}

Communicated by Alberto Calderón, February 28, 1972

Let $K$ be a field of characteristic $\neq 2,3$ and let $\mathfrak{I}_{K}$ be the exceptional Jordan algebra of dimension 27 consisting of hermitian $3 \times 3$ matrices with entries in the Cayley-Dickson algebra $\mathfrak{C}_{K}$. The product $X \circ Y$ in $\mathfrak{I}$ is $\frac{1}{2}(X Y+Y X)$, where $X Y$ is the matrix product. In [3], there are defined a norm (det) and a trace $(\operatorname{tr})$ on $\mathfrak{I}$. Let $(,$,$) be the symmetric trilinear$ form on $\mathfrak{I} \times \mathfrak{I} \times \mathfrak{I}$ such that $(A, A, A)=\operatorname{det}(A)$, and define a bilinear map $\mathfrak{I} \times \mathfrak{I} \rightarrow \mathfrak{I}$, which takes $(A, B)$ to $A \times B$, by requiring that $(A \times B, C)$ $=3(A, B, C)$ for each $C \in \mathfrak{I}$, where $(X, Y)=\operatorname{tr}(X \circ Y)$. Then $A \times A$ plays the role of the matrix adjoint of $A$, and the notions just introduced can be used to define the rank of each element $A \in \mathfrak{I}$. We denote this by $\operatorname{rk}(A)$. In particular, $\operatorname{rk}(A)=3$ if and only if $\operatorname{det}(A) \neq 0$. Let $\mathfrak{f}_{j}=\left\{A \in \mathfrak{I}_{R}: \operatorname{rk}(A)=j\right\}$. The tube domain associated to $\mathfrak{I}$ is

$$
\mathfrak{I}=\left\{Z=X+i Y \in \mathfrak{I}_{c}: Y \in \mathfrak{I}_{3}^{+}\right\},
$$

where $\mathfrak{f}_{j}^{+}=\left\{Y \in \mathfrak{f}_{j}: Y=X^{2}\right.$ for some $\left.X \in \mathfrak{I}_{R}\right\}$.

The group of holomorphic automorphisms of $\mathfrak{I}$ is isogenous to a certain algebraic $Q$-group which is of type $E_{7}$. Baily [1] has defined an arithmetic subgroup $\Gamma$ of $G_{Q}$ which is a unicuspidal subgroup of $G$ and a maximal discrete subgroup of $G_{R}$. Let $J(Z, \gamma)$ be the functional determinant of $\gamma$ at $Z, Z \in \mathfrak{I}$. Let $\Gamma_{0}$ be the subgroup of $\Gamma$ which stabilizes a certain zero-dimensional rational boundary component $\mathfrak{I}_{0}^{\infty}$ of $\mathfrak{T}$, as in $[1, \S 7]$. We let

$$
E_{g}(Z)=\sum_{\gamma_{\epsilon} \Gamma / \Gamma_{0}} J(Z, \gamma)^{g / 18},
$$

where $g \equiv 0(\bmod 36)$ and $g>19$. Then the Eisenstein series $E_{g}$ is an automorphic form of weight $g / 18$ with respect to the group $\Gamma$ and the factor of automorphy $J$. It has an absolutely convergent Fourier expansion

$$
E_{g}(Z)=\sum_{T \in \Lambda^{+}} a_{g}(T) e^{2 \pi i(T, Z)},
$$

AMS 1970 subject classifications. Primary 10D20; Secondary 20G30.

Key words and phrases. Fourier coefficients, Eisenstein series, algebraic $Q$-group of Type $E_{7}$, exceptional Jordan algebra, arithmetic subgroup.

This paper describes a portion of the author's doctoral thesis written under the direction of Professor Walter L. Baily, Jr. at the University of Chicago. 
where $\Lambda^{+}$is the intersection of a certain lattice in $\mathfrak{I}_{R}$ with the set of squares in $\mathfrak{I}_{R}$. The main result of [1] is that $a_{g}(T) \in Q$ for each $T \in \Lambda^{+}$.

For any $T \in \mathfrak{I}_{Q}$ one can define three numerical invariants, the "elementary divisors of $T$." We call their respective $p$-adic orders the " $p$-adic order invariants of $T$." Let $\operatorname{det}_{j}(T)$ be the product of the first $j$ elementary divisors. Then $\operatorname{det}_{3}(T)=\operatorname{det}(T)$ and if $\operatorname{rk}(T)=j$, then $\operatorname{det}_{j}(T) \neq 0$. Let $\Upsilon_{j}$ be the $3 \times 3$ matrix havings 1's in the topmost $j$ positions on the diagonal and zeros elsewhere. The $n$th Bernoulli number $B_{n}$ is defined by the symbolic recursion process $B_{n} \rightarrow B^{n},(1+B)^{n+1}-B^{n+1}=0, B_{0}=1$. In particular, $B_{2 n+1}=0$ if $n \geqq 1$. The purpose of this note is to announce the following result.

THEOREM. For any $T \in \Lambda^{+} \cap \mathfrak{f}_{j}$ with $j=0,1,2,3$,

$$
a_{g}(T)=a_{g}\left(\Upsilon_{j}\right) \operatorname{det}_{j}(T)^{g+3-4 j} \prod_{p \mid \operatorname{det}_{j}(T)} f_{T}^{p}\left(p^{4 j-3-g}\right),
$$

where

$$
a_{g}\left(\Upsilon_{j}\right)=2^{j(2 j-1)} \prod_{n=0}^{j-1}\left\{\frac{g-4 n}{B_{g-4 n}}\right\}
$$

and where $f_{T}^{p}$ is a monic polynomial with rational integer coefficients and with degree $D=\operatorname{ord}_{p}\left(\operatorname{det}_{j}(T)\right)$. Furthermore, $f_{T}^{p}$ is determined by the $p$-adic order invariants of $T$; hence, for fixed $g, a_{g}(T)$ depends only on the elementary divisors of $T \in \Lambda^{+}$.

Let \|\|$_{p}$ be the ordinary $p$-adic absolute value. Then $\left\|\operatorname{det}_{j}(T)\right\|_{p}^{4 j-3-g_{f}} f_{T}^{p}\left(p^{4 j-3-g}\right)$ is a rational integer. The Fourier coefficients $a_{g}(T)$, for fixed $g$, are integral multiples of $a_{g}\left(\Upsilon_{j}\right)$, where $j=\operatorname{rk}(T)$. Note that $a_{g}\left(\Upsilon_{j}\right) \in \boldsymbol{Q}$.

COROLlaRY. Let $\delta_{g}$ be the product of the numerators of the rational numbers $B_{g-4 n}$, where $n=0,1,2$. Then the $\Gamma$-automorphic form $\delta_{g} E_{g}$ has rational integer Fourier coefficients.

Suppose that $T \in \Lambda^{+} \cap \mathfrak{f}_{2}$ and that the order invariants of $T$ are $\tau, \tau^{\prime}$ where $\tau \leqq \tau^{\prime}$. Then $f_{T}^{p}(X)=\sum_{k=0}^{\tau} p^{4 k} \sum_{m=k}^{\tau+\tau^{\prime}-k} X^{m}$. We have not determined $f_{T}^{p}$ so explicitly when $\operatorname{rk}(T)=3$, but it is easy to compute individual examples from our work. For example, when $T=p \Upsilon_{3}$, we have

$$
f_{T}^{p}(X)=X^{3}+\left(p^{8}+p^{4}+1\right) X^{2}+\left(p^{8}+p^{4}+1\right) X+1
$$

Similar but essentially less precise results have been obtained in the case of the group $S p_{n}(Z)$ acting on the Siegel upper half-space $\mathfrak{S}_{n}$ of rank $n$ by Maass [4] when $n=2$, by Siegel [5], and by Eichler [2]. Both Maass 
and Eichler used the theory of Hecke operators, while Siegel relied on the analytic theory of quadratic forms. By contrast, our methods are entirely elementary.

\section{REFERENCES}

1. W. L. Baily, Jr., An exceptional arithmetic group and its Eisenstein series, Ann. of Math. (2) 91 (1970), 512-549. MR 42 \#4674.

2. M. Eichler, Zur Begründung der Theorie der automorphen Funktionen in mehreren Variablen, Aequationes Math. 3 (1969), 93-111. MR 41 \#2056.

3. H. Freudenthal, Zur ebenen Oktavengeometrie, Nederl. Akad. Wetensch. Proc. Ser. A 56 = Indag. Math. 15 (1953), 195-200. MR 15, 56.

4. H. Maass, Die Fourierkoeffizienten der Eisensteinreihen zweiten Grades, Mat.-Fys. Medd. Danske Vid. Selsk. 34 (1964), no. 7, 25 pp. MR 30 \#1985.

5. C. L. Siegel, Über die Fourierschen Koeffizienten der Eisensteinschen Reihen, Mat.Fys. Medd. Danske Vid. Selsk. 34 (1964), no. 6, 20 pp. MR 30 \#1984.

Department of Mathematics, University of Chicago, Chicago, Illinois 60637

Current address: School of MAThematics, Institute for AdVANCEd Study, Princeton, NeW JERSEY 08540 